\title{
Sedimentation and multiphase equilibria in suspensions of colloidal hard rods
}

\author{
S. V. Savenko and Marjolein Dijkstra \\ Debye Institute, Soft Condensed Matter Group, Utrecht University, Princetonplein 5, 3584 CC Utrecht, The Netherlands
}

(Received 6 July 2004; published 11 November 2004)

\begin{abstract}
Sedimentation and multiphase equilibria in a suspension of hard colloidal rods are explored by analyzing the (macroscopic) osmotic equilibrium conditions. We observe that gravity enables the system to explore a whole range of phases varying from the most dilute phase to the densest phase, i.e., from the isotropic $(I)$, to the nematic $(N)$, to the smectic $(\mathrm{Sm})$, to the crystal $(K)$ phase. We determine the phase diagrams for hard spherocylinders with a length-to-diameter ratio of 5 for a semi-infinite system and a system with fixed container height using a bulk equation of state obtained from simulations. Our results show that gravity leads to multiphase coexistence for the semi-infinite system, as we observe $I, I+N, I+N+\mathrm{Sm}$, or $I+N+\mathrm{Sm}+K$ coexistence, while the finite system shows $I, N, \mathrm{Sm}, K, I+N, N+\mathrm{Sm}, \mathrm{Sm}+K, I+N+\mathrm{Sm}, N+\mathrm{Sm}+K$, and $I+N+\mathrm{Sm}+K$ phase coexistence. In addition, we compare our theoretical predictions for the phase behavior and the density profiles with Monte Carlo simulations for the semi-infinite system and we find good agreement with our theoretical predictions.
\end{abstract}

DOI: 10.1103/PhysRevE.70.051401

PACS number(s): 82.70.Dd, 64.70.Md

\section{INTRODUCTION}

Gravity is often non-negligible in colloidal suspensions, as the gravitational energy becomes comparable to the thermal energy for colloid sizes of about a micrometer. Hence, a spatial inhomogeneous suspension is obtained due to the gravitational field, which is characterized by a density profile $\rho(z)$ that varies with altitude $z$. The parameter that is associated with a gravitational field is the so-called gravitational length and reads $\ell=(\beta m g)^{-1}$ where $m$ is the effective or buoyancy mass of the colloidal particle, $\beta=\left(k_{B} T\right)^{-1}$ the inverse temperature with $k_{B}$ Boltzmann's constant, and $g$ the gravitational acceleration. Typically, $\ell$ is of the order of micrometers to millimeters for colloidal particles. The density profile $\rho(z)$ follows from a competition between minimal energy (all colloids at the bottom) and maximum entropy (a homogeneous distribution in the available volume). In the case of a very dilute colloid concentration or at high altitude, where the suspension becomes sufficiently dilute, the system behaves like an ideal gas and the system obeys the Boltzmann distribution, yielding an exponential density profile with a decay length given by $\ell$. In 1910, Jean Perrin measured such a density profile under the microscope which enabled him to determine Boltzmann's constant and hence Avogadro's number [1]. However, when the interactions become important, the density profile becomes highly nonexponential. Density profiles have been calculated for suspensions of hard and charged colloidal spheres using density functional theory [2], and are measured by light scattering techniques [3]. The measured concentration profiles can be inverted to obtain the osmotic equation of state [2-4]. Nontrivial sedimentation profiles have been predicted recently for charged colloids [5-9] (and measured experimentally [10]), colloid-polymer mixtures [11-14], and binary hardsphere mixtures [15].

In this paper we consider suspensions of hard rods, which serve as a simple model for colloidal rodlike particles, like the tobacco mosaic virus, fd virus, and boehmite or silica rods. The bulk phase behavior of hard-rod fluids has been studied thoroughly in many theoretical and simulation studies [16-18]. Suspensions of colloidal rods show lyotropic liquid crystalline behavior and form isotropic, nematic, smectic, and crystal phases upon increasing the concentration. Despite the fact that gravity is often non-negligible for colloidal rods, there are only a few theoretical studies that include its effect on the phase behavior [19] and on the structure of the fluid [20]. In this paper we determine the phase diagram and density profiles from macroscopic equilibrium conditions using the bulk equation of state of hard spherocylinders with a length-to-diameter ratio $L / D=5$ obtained from Monte Carlo simulations [21]. We consider two situations: (1) a semi-infinite system that extends to infinity in the vertical $z$ direction and (2) a finite system. References $[19,20]$ were both focused on the finite system, while disregarding the situation of a semi-infinite system. Surprisingly, the phase behavior depends drastically on the boundary conditions of the system. Finally, we compare our results derived from macroscopic equilibrium conditions directly with Monte Carlo simulations for the semi-infinite system. Moreover, we examine the accuracy of the osmotic equation of state derived from an inversion of the sedimentation profiles of hard spherocylinders.

The paper is organized as follows. In Sec. II we describe the model and the macroscopic description of sedimentation equilibria. In Sec. III, we determine phase diagrams for the finite and semi-infinite systems. We present Monte Carlo simulation results in Sec. IV and we end with some concluding remarks in Sec. V.

\section{MACROSCOPIC DESCRIPTION OF SEDIMENTATION EQUILIBRIUM}

We consider a system of hard spherocylinders with a length-to-diameter ratio of $L / D$ suspended in a solvent. The suspending solvent is regarded as an incompressible structureless continuum, characterized by its mass density $\widetilde{\rho}$. The 
effective or buoyancy mass of a spherocylinder is, according to Archimedes' principle,

$$
m=m_{0}-\tilde{\rho} v,
$$

where $m_{0}$ is the bare mass of the rod and $v=\pi\left(L D^{2} / 4\right.$ $\left.+D^{3} / 6\right)$ the volume of the rod. In a gravitational field oriented along the $z$ direction of the vessel, the rods are subjected to the external potential

$$
\phi(z)=m g z,
$$

where $z$ is the vertical coordinate, and $g$ is the gravitational acceleration.

When the density does not vary rapidly with the height of the sample, which is usually the case if the particles are not too large and heavy, the macroscopic description of the system is applicable and the equilibrium condition reads [22]

$$
\frac{d P(z)}{d z}=-m g \rho(z),
$$

where $P$ is the osmotic pressure.

Equation (3) allows us to determine the equation of state of the system from a single measurement of the concentration profile, which is convenient in experimental or simulation studies $[2,4]$. The pressure at arbitrary height $z$ can be obtained using

$$
\beta P(z)=\beta P(0)-\ell^{-1} \int_{0}^{z} d z^{\prime} \rho\left(z^{\prime}\right)
$$

where $\beta=\left(k_{B} T\right)^{-1}$ and $\ell=(\beta m g)^{-1}$. If the concentration profile $\rho(z)$ is measured, elimination of $z$ between $\rho(z)$ and $P(z)$ leads directly to the osmotic equation of state $P(\rho)$ of the colloidal suspension.

On the other hand, if the temperature is assumed to be constant throughout the sample, the pressure depends only on the local density and Eq. (3) can be rewritten as a nonlinear differential equation for $\rho(z)$,

$$
\frac{d \rho(z)}{d z}=-\frac{\chi_{T}(\rho) \rho(z)}{\ell}
$$

where $\chi_{T}=(\partial \beta P / \partial \rho)_{T}^{-1}$ is the reduced osmotic compressibility of the bulk fluid at density $\rho$. If the osmotic equation of state $P(\rho)$ is known explicitly, the density profile at various values for the gravitational length can be obtained from Eq. (5).

\section{PHASE DIAGRAMS FOR COLLOIDAL RODS IN A GRAVITATIONAL FIELD}

We determine phase diagrams for colloidal rods in a gravitational field using the macroscopic conditions for sedimentation equilibrium. We model the colloidal rods as hard spherocylinders, for which the bulk phase diagram is well explored $[18,21]$. The phase diagram shows an isotropic fluid phase $(I)$, nematic $(N)$ and smectic $A(\mathrm{Sm})$ phases, and a crystalline phase $(K)$. In order to determine the phase behavior for colloidal rods in a gravitational field, we employ fits to the equation of states obtained from Monte Carlo simula-

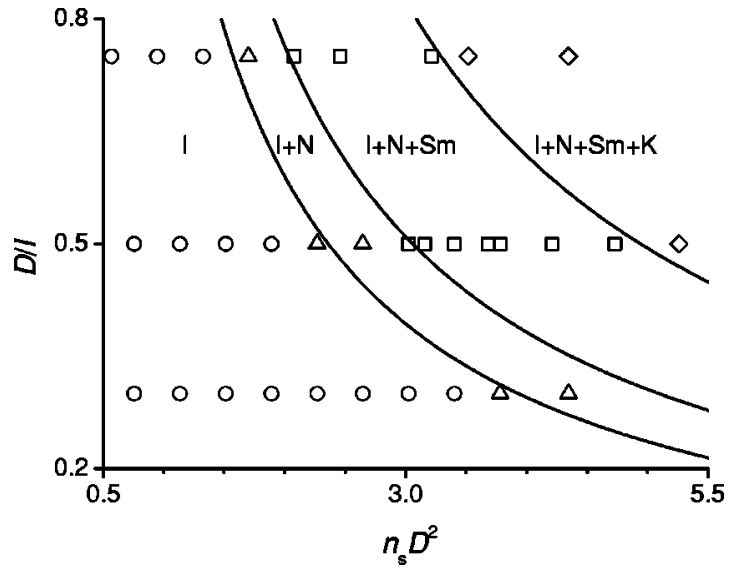

FIG. 1. Phase diagram for hard spherocylinders (colloidal rods) with $L / D=5$ in a gravitational field for a semi-infinite system. We plot the gravitational length of the rods $D / \ell$ versus the number of rods per unit surface $n_{s} D^{2}$. The symbols denote the state points employed in our simulations of spherocylinders. $\bigcirc$ denotes the isotropic phase, $\triangle$ the isotropic-nematic $(I+N)$ coexistence, $\square$ the isotropic-nematic-smectic- $A(I+N+\mathrm{Sm})$ coexistence, and $\diamond$ the isotropic-nematic-smectic- $A$-crystal $(I+N+\mathrm{Sm}+K)$ coexistence.

tions of hard spherocylinders with a length-to-diameter ratio $L / D=5$ [21]. We determine the phase diagrams for two situations: (1) a semi-infinite system, and (2) a finite system. We show that the phase behavior depends sensitively on the boundary conditions of the system. Below, we discuss the two situations in more detail.

\section{A. Semi-infinite system}

We first consider the case of a semi-infinite system extending from $z=0$ to $\infty$. We confine $N$ rods in an open rectangular vessel with a horizontal $x y$ cross section of area $S$. The bottom of the system is located at $z=0$ and vertical confinement is determined by the gravitational force exerted on the particles. We determine the phase behavior using the values for the pressure at phase coexistence determined by previous simulations in Ref. [21]. Direct integration of Eq. (3) using the boundary condition $\int_{0}^{\infty} d z \rho(z)=N / S \equiv n_{s}$, i.e., keeping the number of particles per unit surface fixed, and employing $P(z=\infty)=0$, yields a simple relation for the pressure at $z=0$, i.e., at the bottom of the sample,

$$
P(z=0)=m g n_{s} .
$$

Equating the pressure at phase coexistence with the pressure at the bottom of our sample, given by Eq. (6), we can determine easily the phase boundaries of our system, i.e., which phase appears at the bottom of our system followed by the more dilute phases at higher altitudes. In Fig. 1, three phase boundaries denoted by the solid lines are shown in the phase diagram for a semi-infinite system. At low gravity, i.e., low values of $D / \ell$, and low $n_{s} D^{2}$, we observe an isotropic phase throughout the whole system. This regime is denoted by $I$ in our phase diagram. At higher gravity or $n_{s}, P(z=0)$ becomes larger than $P_{I N}$, i.e., the pressure at $I-N$ coexistence, and we observe a nematic phase at the bottom of our sample. At 


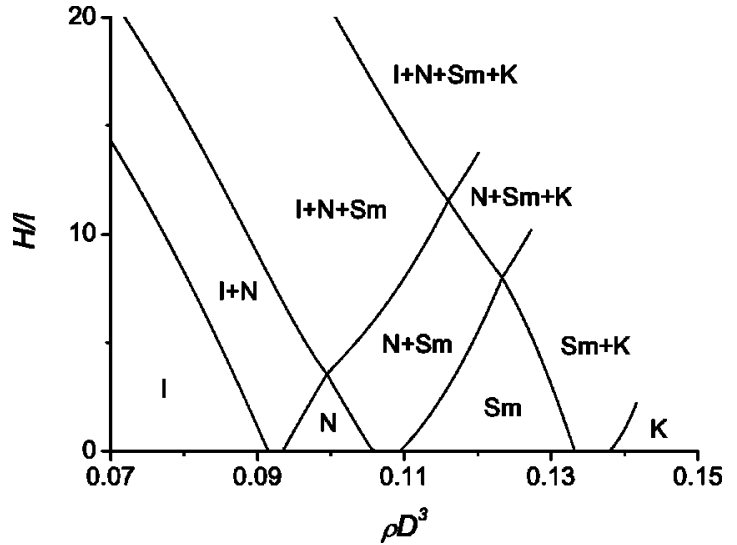

FIG. 2. Phase diagram for hard spherocylinders (colloidal rods) with $L / D=5$ in a gravitational field for a closed vessel. We plot the reduced sample height $H / \ell$ with $\ell$ the gravitational length of the rods versus the dimensionless rod density $\rho D^{3}$.

higher altitudes, we still observe an isotropic phase as the system becomes more and more dilute at higher $z$. Hence, we observe two phases simultaneously in our sample with the denser phase $(N)$ at the bottom and the isotropic phase at the top. When $P(z=0)$ becomes larger than the pressure $P_{N \text { Sm }}$ at $N$-Sm coexistence, we find three-phase coexistence with the smectic phase at the bottom of the container followed by the nematic and the isotropic phases at higher altitudes. For $P(z=0)>P_{\text {Sm } K}$, four-phase coexistence is predicted with the crystal phase at $z=0$. The pressure at $\mathrm{Sm}-K$ coexistence is denoted by $P_{\mathrm{Sm} K}$. It is worth mentioning that the phase diagram shows one-, two-, three-, and four-phase coexistence regions and that the isotropic phase is always present in a semi-infinite system at sufficiently high altitudes.

\section{B. Finite system}

In the previous subsection we considered the situation of a suspension in an open vessel unlimited in the $z$ direction, which becomes infinitely dilute in the limit of $\ell \rightarrow \infty$, i.e., no gravity. In this section, we consider a suspension of $N$ colloidal rods confined in all directions to a volume $V$. The bottom of the system is again located at $z=0$, while the height of the vessel $H$ is fixed. In the absence of gravity, the suspension is homogeneously distributed with a uniform density $N / V$. Employing Eq. (5) using the boundary condition $\int_{0}^{H} d z \rho(z) / H=N / V$ yields the density at the bottom $\rho(z$ $=0)$ and at the top $\rho(z=H)$ of the sample from which we can determine the phase diagram. Figure 2 shows the phase diagram in the reduced sample height $\mathrm{H} / \ell$-dimensionless rod density $\rho D^{3}$ representation. On the horizontal axis we find bulk coexistence densities of the isotropic-nematic, nematicsmectic, and smectic-crystal transitions for zero gravity, i.e., $H / \ell=0$. At sample heights of about three times the gravitational length, we observe an extremely rich phase behavior. For instance, at rod densities $\rho D^{3} \sim 0.1$, we find a pure nematic phase at low sample heights, which is sandwiched by a more dilute phase $(I)$ at the top and a denser phase $(\mathrm{Sm})$ at the bottom, as soon as the sample height exceeds $H / \ell$ $=3.55$. The resulting sample exhibits three-phase coexistence of isotropic, nematic, and smectic phases. At rod densities $\rho D^{3} \sim 0.125$, a pure smectic phase can be transformed in a three-phase $(N+\mathrm{Sm}+K)$ coexistence by increasing the sample height. On the other hand, at sample heights $H / \ell$ $\sim 3$, a single $I$ phase can be transformed upon increasing the rod density to a two-phase $(I+N)$ coexistence, a single $N$ phase, $N+\mathrm{Sm}$ coexistence, a single $\mathrm{Sm}$ phase, $\mathrm{Sm}+K$ coexistence, to a single crystal phase. At sample heights $H / \ell$ $\sim 10$ the sequence is $I, I+N, I+N+\mathrm{Sm}, N+\mathrm{Sm}, N+\mathrm{Sm}$ $+K, \mathrm{Sm}+K, K$, upon increasing the rod density. Four-phase coexistence is observed at rod densities $\rho D^{3} \sim 0.115$ at sample heights more than about 11 gravitational lengths. It is worth noting that there are striking differences with the semiinfinite system. When phase coexistence is observed in the semi-infinite system, the most dilute phase at sufficiently high altitudes is always the isotropic phase. This is not the case in the finite system, yielding many more possibilities for multiphase coexistence and resulting in a much more complicated phase diagram as shown in Fig. 2 compared to the semi-infinite system in Fig. 1.

\section{SIMULATIONS}

In this section, we present a Monte Carlo study of a system of hard spherocylinders with a length-to-diameter ratio $L / D=5$ in a gravitational field. Each spherocylinder consists of a cylindrical part with diameter $D$ and a length $L$ with spherical caps of diameter $D$ at both ends. The bulk phase diagram of hard spherocylinders is well explored [18,21] and shows a rich phase behavior including isotropic, nematic, smectic $A$, and crystalline phases. In Refs. [23-25], the interfacial behavior of a hard-rod fluid is investigated using density functional theory and computer simulations. The authors find complete wetting of the nematic phase at the wallisotropic fluid interface and a uniaxial to biaxial surface transition well below the bulk $I-N$ transition. Moreover, when the hard-rod fluid is confined by two walls, a significant shift of the $I-N$ transition is found to lower densities compared to bulk. In this section, we study the effect of a gravitational field on the phase behavior of hard rods using computer simulations. We compare our theoretical predictions based on the macroscopic equilibrium conditions on the phase diagram with simulation results. We study a system with a fixed number of hard spherocylinders $N$ in a semi-infinite box with lateral dimensions $L_{x}$ and $L_{y}$ applying periodic boundary conditions in the $x$ and $y$ directions. The $z$ dimension of the box is infinite. While at $z=0$ a planar hard wall is located, the rods are free to move upward. The confinement of the system is implemented by a gravitational field along the $z$ direction. Each rod is subjected to the external potential $\phi(z)=m g z$ with $m$ the buoyancy mass, $g$ the acceleration of gravity, and $z$ the height of the center of mass of the rod. The number of particles and the dimensions of the box are varied in the simulations to adjust the pressure at the bottom of the system. The starting configuration is prepared as follows. A close-packed face-centered-cubic (fcc) lattice of spheres of diameter $D$ with its (001) plane in the $x y$ plane was stretched in the $x$ direction by a factor of $L / D+1$ in order to accommodate the close-packed crystal of spherocylinders. This lat- 


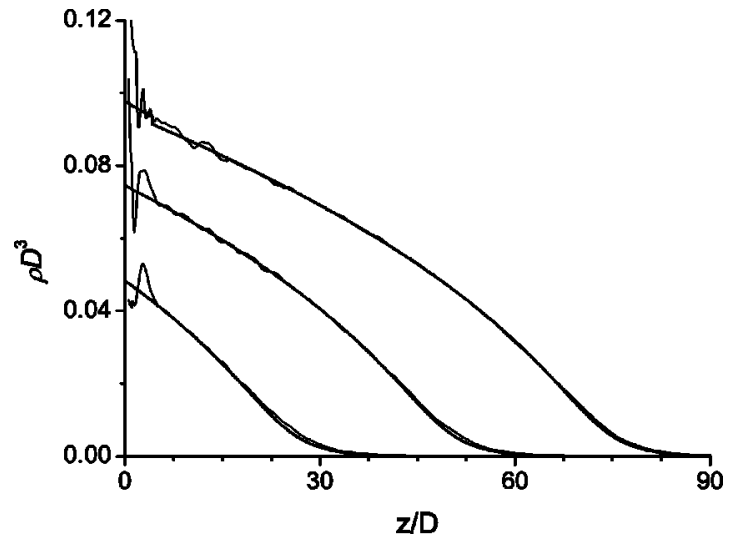

FIG. 3. Density profiles $\rho D^{3}$ versus $z / D$ for a semi-infinite system with inverse gravitational length $D / \ell=0.30$, and for varying number of particles per unit surface $n_{s} D^{2}=0.756,2.268,4.348$ (from bottom to top). The thin lines are the coarse-grained density profiles from computer simulations, while the thick lines are those obtained from Eq. (5) using the equation of state of Ref. [21].

tice was subsequently expanded in the $x$ and $y$ directions. For inhomogeneous phases, such as the smectic and crystal phases, it is often essential that the simulation box can change its size (and maybe its shape), in order to accommodate the changes in the phase structures without creating high stresses in the sample. However, as the concentration varies with height in a gravitational field, it is impossible to avoid stresses at each height $z$. We have chosen the area of the sample such that the phase at the bottom is well accommodated or is commensurate with the dimensions of the cross section of the container. We check for equilibrium by monitoring the height of the center of mass of the system. When equilibrium is reached we perform sampling at $\sim 1.5 \times 10^{6}$ Monte Carlo cycles (one cycle is one attempted move per particle), the profiles are sampled in bins of width $0.1 D$. The sampled quantities are the density profile and the profiles of the eigenvalues of the standard $3 \times 3$ nematic order parameter tensor defined as [26]

$$
Q_{\alpha \beta}\left(z_{j}\right)=\frac{1}{n_{j}}\left\langle\sum_{i=1}^{n_{j}} \frac{3 u_{\alpha}^{i} u_{\beta}^{i}-\delta_{\alpha \beta}}{2}\right\rangle
$$

where $u_{\alpha}^{i}$ is the $\alpha$ component of the unit orientation vector of particle $i$ with $\alpha=x, y, z$, and $n_{j}$ is the number of particles present in bin $j$. The Kronecker delta is $\delta_{\alpha \beta}$. Diagonalizing this tensor gives three orientational ordering eigenvalues $\lambda_{+}, \lambda_{0}$, and $\lambda_{-}$for each bin.

We perform simulations of spherocylinders with $L / D=5$ at three values of the inverse gravitational length $D / \ell=0.3$, 0.5 , and 0.75 , and for varying number of particles per unit surface $n_{s} D^{2}$. The explicit values for $D / \ell$ and $n_{s} D^{2}$ which are employed in the simulations are displayed by the dots in Fig. 1. We display coarse-grained density profiles for $D / \ell=0.30$ and 0.75 for varying values of $n_{s} D^{2}$ in Figs. 3 and 4. Similar results were obtained for $D / \ell=0.50$. We show plots for only a few values of $n_{s} D^{2}$ for clarity. At high altitudes (large $z$ ) we find that the density tends to zero, while the highest density is observed at the bottom of the sample as expected. For

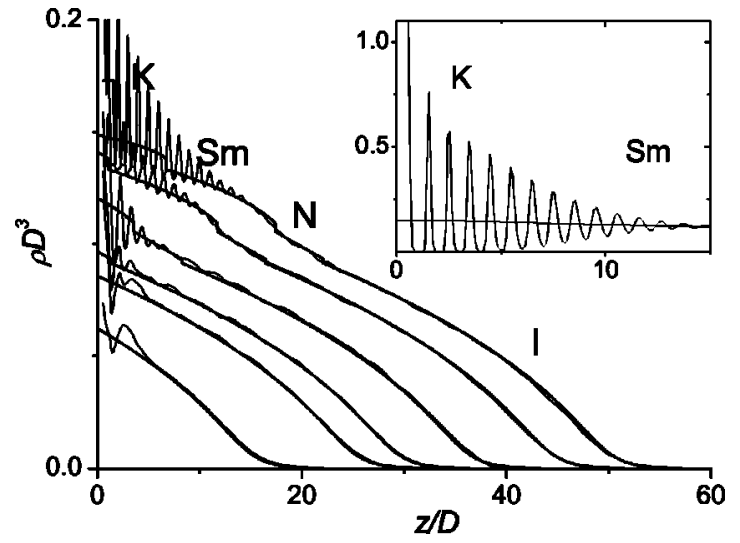

FIG. 4. Density profiles $\rho D^{3}$ versus $z / D$ for a semi-infinite system with inverse gravitational length $D / \ell=0.75$, and for varying number of particles per unit surface $n_{s} D^{2}$ of $0.567,1.323,1.701,2.457,3.51,4.34$ (from bottom to top). The thin lines are the coarse-grained density profiles from computer simulations, while the thick lines are those obtained from Eq. (5) using the equation of state of Ref. [21]. The inset shows the raw density profile from simulations for $n_{s} D^{2}=4.34$.

comparison, we also plot the density profiles obtained from Eq. (5) and employing the equation of state from Ref. [21]. We find good agreement of the simulation results with the theoretical predictions based on the macroscopic osmotic equilibrium conditions. We, therefore, believe that also the theoretical predictions on the phase behavior are reliable. The density fluctuations due to the ordering of the rods induced by the wall or due to the ordering in the smectic and crystal phase is, of course, missing in the density profiles obtained from the macroscopic osmotic equilibrium conditions. We wish to mention that the phase at the bottom of the container, and hence the phase diagram can be determined by comparing the theoretical or simulation contact densities at $z=0.5 D$ with the bulk coexisting densities. The coexisting densities are determined by McGrother et al. using computer simulations [21]. The reduced densities are at $I-N$ coexistence $\rho D^{3}=0.0914(I)$ and $\rho D^{3}=0.0932(N)$, at $N$-Sm coexistence $\rho D^{3}=0.1061(N)$ and $\rho D^{3}=0.1094(\mathrm{Sm})$, and at Sm- $K$ coexistence $\rho D^{3}=0.1319(\mathrm{Sm})$ and $\rho D^{3}=0.1380(K)$ [21]. For the two lowest density profiles in Figs. 3 and 4, we find that the contact density is lower than the coexisting density of the nematic phase at $I-N$ coexistence; hence, the whole sample is in the isotropic phase in agreement with the theoretical phase diagram in Fig. 1. The contact densities for $n_{s} D^{2}=4.348$ in Fig. 3 and $n_{s} D^{2}=1.701$ in Fig. 4 are higher than the coexisting density of the nematic phase at $I-N$ coexistence, but lower than that at $\mathrm{N}$-Sm coexistence, and we find two-phase coexistence of the isotropic phase and the nematic phase. We find that the contact density for $n_{s} D^{2}=2.457$ in Fig. 4 is higher than the coexisting density of the smectic phase at $N$-Sm coexistence, but lower than that at $\mathrm{Sm}-K$ coexistence. For this state point, we observe three-phase coexistence of smectic, nematic, and isotropic phases. For all these density profiles, we find good agreement with the theoretical predictions for the phase behavior, as one might expect on the basis of the good agreement of the density profiles with the theo- 

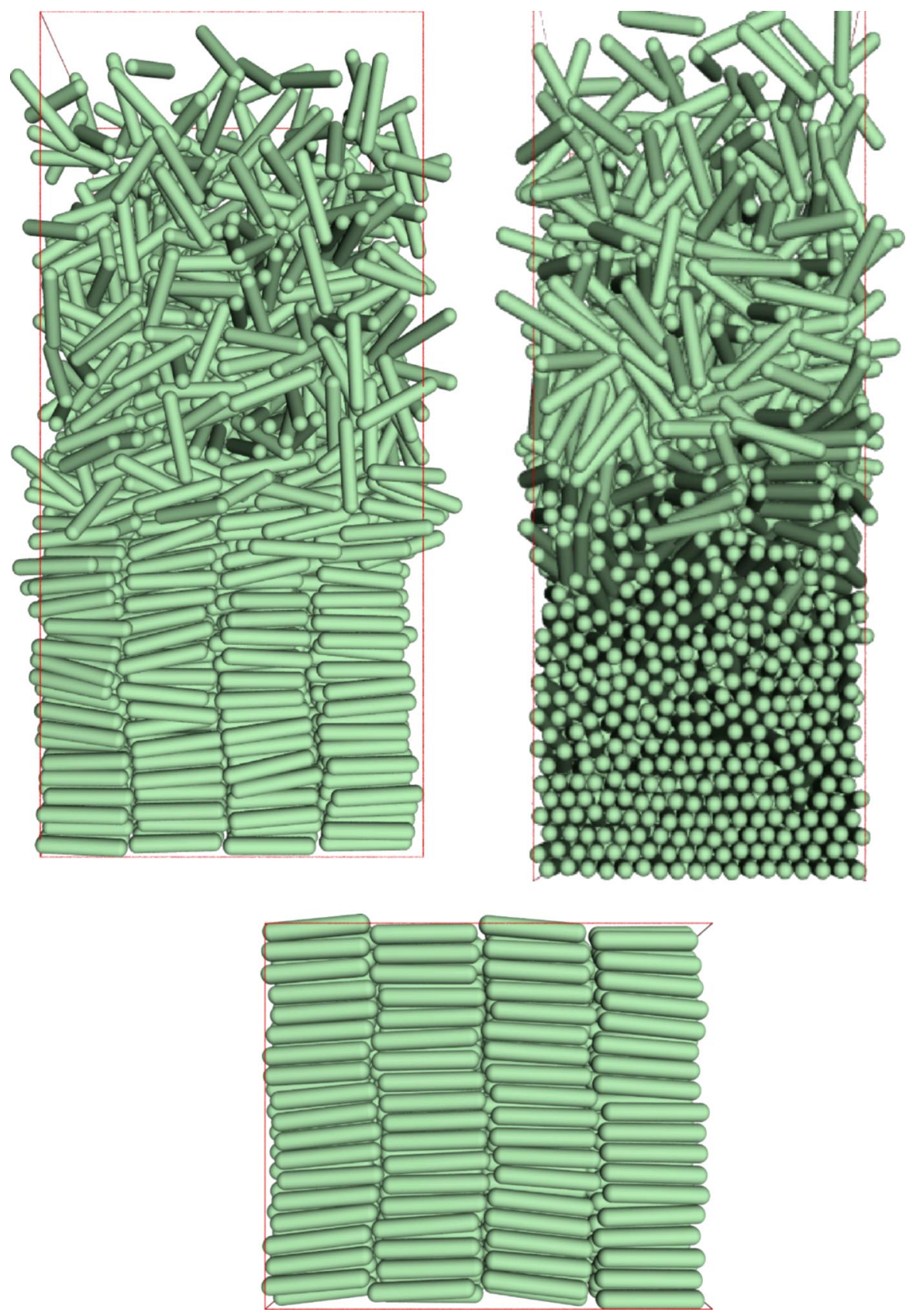

FIG. 5. Snapshots of typical configurations, two sideviews (a) and (b), and a bottom view (c), obtained from simulations of hard spherocylinders with $L / D=5$, for number of particles per unit surface $n_{s} D^{2}=4.34$ and for an inverse gravitational length $D / \ell=0.75$.

retical ones. Closer inspection of Fig. 1 shows that the $I+N$ coexistence starts at lower $n_{s} D^{2}$ for $D / \ell=0.5$ and 0.3 compared with the theoretical predictions. A similar shift was also found in previous simulations of a hard-rod fluid in contact with a planar hard wall: the uniaxial to biaxial surface transition occurs well below the bulk $I-N$ transition [23-25]. However, it is impossible to determine the contact density from the density profiles obtained from simulations at high $n_{s} D^{2}$ due to the large density fluctuations close to the wall. Even coarse-graining the density profiles does not give us a sufficiently accurate value for the contact density. However, the appearance of the crystal phase can be observed in the raw (not coarse-grained) density profiles. The inset of Fig. 4 shows the raw density profile for $n_{s} D^{2}=4.34$. We clearly observe the formation of crystalline layers at the bottom and smectic layers at higher altitudes: the density drops to zero between the density peaks close to the wall (crystal phase), while only pronounced peaks are observed at higher $z$ (smectic phase). The system is in the four-phase coexistence region for $n_{s} D^{2}=4.34$ and $D / \ell=0.75$. This multiphase coexistence can also be appreciated from the snapshots in Fig. 5 that shows nice crystalline ordering at the bottom of the container in Fig. 5(c) and hexagonal ordering in Fig. 5(b). At higher altitudes, the hexagonal ordering is destroyed, and a smectic phase appears. The smectic layers disappear at even higher heights as can be seen in Fig. 5(a), and we observe a nematic phase. At high altitudes, we observe the isotropic phase. 

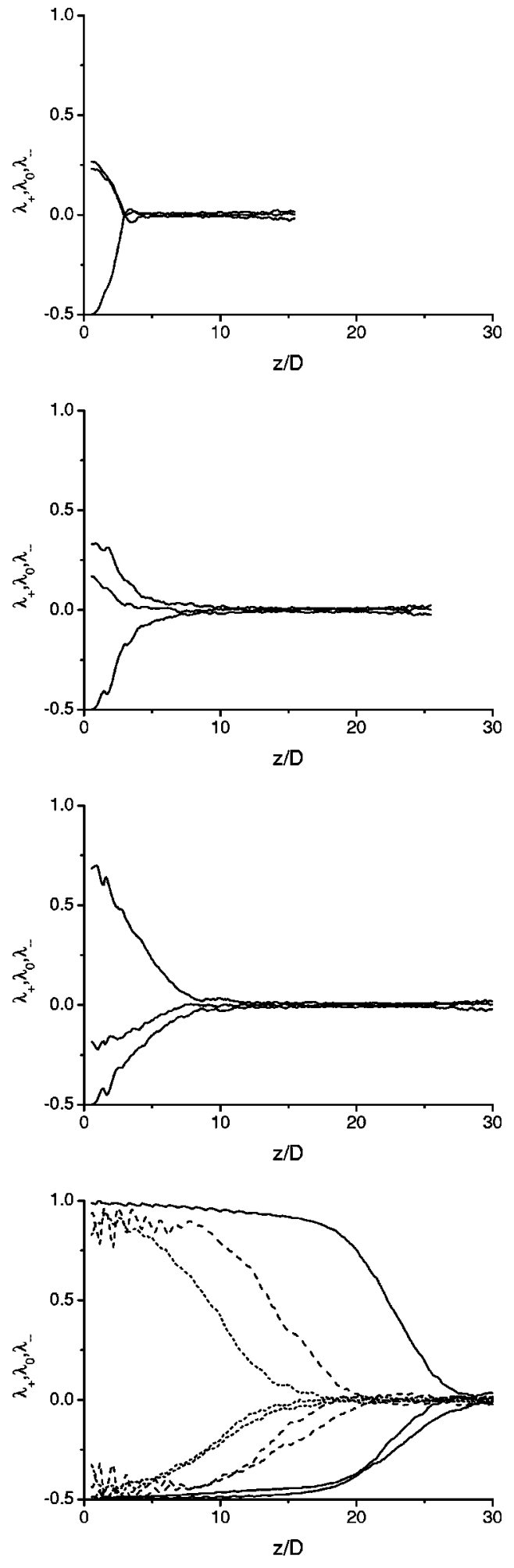

FIG. 6. The profiles of the eigenvalues $\lambda_{+}, \lambda_{0}$, and $\lambda_{-}$of the nematic order parameter tensor (7) versus $z / D$ for a semi-infinite system with inverse gravitational length $D / \ell=0.75$, and varying number of particles per unit surface (a) $n_{s} D^{2}=0.567$, (b) $n_{s} D^{2}$ $=1.323$, (c) $n_{s} D^{2}=1.701$, and (d) $n_{s} D^{2}=2.457$ (short-dashed), 3.51 (dashed), 4.34 (solid).

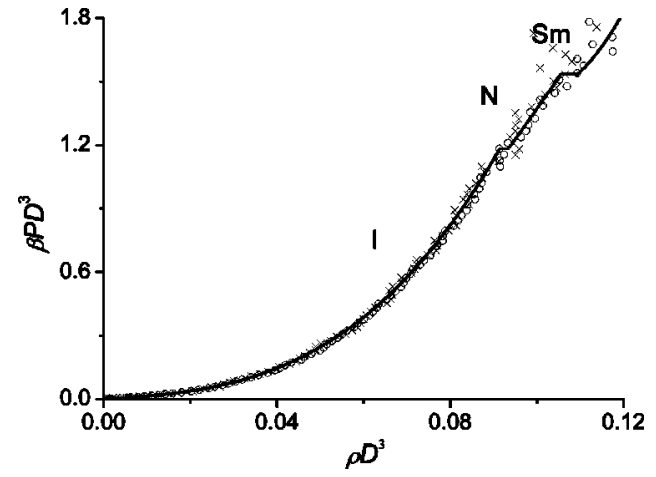

FIG. 7. Equation of state of hard spherocylinders with a lengthto-diameter ratio $L / D=5$ obtained by inverting the coarse-grained density profiles from Fig. 4 versus reduced densities $\rho D^{3}$ for $n_{s} D^{2}$ $=3.21(\times)$ and $n_{s} D^{2}=4.34(\bigcirc)$, while - represents the bulk equation of state from Ref. [21].

In Fig. 6, we plot the order parameter profiles for $D / \ell$ $=0.75$ and varying values of $n_{s} D^{2}$. In bulk, the value of the largest eigenvalue of this tensor determines the nematic ordering, which is zero for an isotropic fluid phase and 1 for perfectly aligned rods. In the presence of the wall at the bottom of the sample the lowest eigenvalue of this tensor approaches $-1 / 2$ as the rods close to the wall have to be oriented parallel to the surface. Consequently, the two largest eigenvalues are equal to $1 / 4$ when there is no in-plane order; see Fig. 6 (the short-dashed lines for $n_{s} D^{2}=0.567$ ). The onset of biaxial and nematic ordering at the bottom can be observed from the difference of the two largest eigenvalues, which shows the appearance of a preferred in-plane orientation (the dash-double-dotted lines for $n_{s} D^{2}=1.323$ ). As $n_{s} D^{2}$ increases this difference increases. The nematic phase appears at the bottom of the container when the largest eigenvalue exceeds 0.5 at $z=0$ (dash-dotted line for $\left.n_{s} D^{2}=1.701\right)$, which corresponds well with the value of the nematic order parameter of 0.471 for the nematic phase at $I-N$ coexistence [21]. Three-phase coexistence is observed when the largest eigenvalue is roughly 0.9 at the bottom (dotted and dashed lines for $n_{s} D^{2}=2.457$ and 3.51 , respectively), again in agreement with the fact that the nematic order parameter of the smectic phase in bulk is about 0.893 at $N$-Sm coexistence [21]. A crystal phase (solid line for $n_{s} D^{2}=4.34$ ) is found at the bottom of the container when the largest eigenvalue approaches 1, while the order parameter of the crystal phase in bulk is about 0.974 at $\mathrm{Sm}-K$ coexistence [21].

Finally, we obtain the osmotic equation of state for hard spherocylinders by inverting the coarse-grained simulation sedimentation profiles using Eq. (4) and by eliminating $z$ between $\rho(z)$ and $P(z)$. In Fig. 7, we show the results using the profiles for inverse gravitational length $D / \ell=0.75$ and number of particles per unit surface $n_{s} D^{2}=3.21$ and 4.34. For comparison, we also plot the osmotic equation of state obtained from bulk simulations performed at many different densities of Ref. [21] and we find good agreement, even for densities higher than those at $I-N$ coexistence. Similar good agreement of the equation of state of the isotropic and of the nematic phase was also found by inverting sedimentation profiles for $D / \ell=0.30$ and 0.50 , and other values of $n_{s} D^{2}$ that we considered. 


\section{CONCLUSIONS}

We have investigated sedimentation and phase equilibria in suspensions of hard spherocylinders with a length-todiameter ratio $L / D=5$ by analyzing the (macroscopic) osmotic equilibrium conditions. We present phase diagrams for a semi-infinite system and for a finite system. We find that the phase behavior depends in great detail on the boundary conditions of the system. To the best of our knowledge, we believe that this is the first study that investigates the dependence of the phase behavior on the boundary conditions of the system. In addition, we compare our theoretical predictions for the phase behavior and sedimentation profiles with Monte Carlo simulations for the semi-infinite system. We find very good agreement between the two sets of results. Moreover, we find excellent agreement of the osmotic equation of state obtained from inverting the coarse-grained sedimentation profiles from a single simulation with the bulk equation of state determined from bulk simulations at many different densities [21], even for densities in the nematic phase. This surprisingly good agreement for densities beyond $I-N$ coexistence can be understood as the interfacial width of the isotropic-nematic interface is very small for the gravitational lengths considered in this work.

It is interesting to study the effect of the addition of an extra component to the sedimentation profiles of a suspension of hard rods, e.g., a nonadsorbing polymer that might yield a floating liquid phase similar to that found in Refs. $[13,14]$, or thinner rods that might give a nontrivial density profile of floating thick rods in a suspension of thin rods similar to that in Ref. [15]. We plan to study the sedimentation profiles of colloidal rod mixtures in future work.

\section{ACKNOWLEDGMENTS}

We thank René van Roij for stimulating discussions and we wish to thank the Dutch National Computer Facilities foundation for access to the SGI Origin3800. The High Performance Computing group of Utrecht University is gratefully acknowledged for ample computer time.
[1] J. Perrin, J. Phys. 9, 5 (1910).

[2] T. Biben, J. Hansen, and J. Barrat, J. Chem. Phys. 98, 7330 (1993).

[3] R. Piazza, T. Bellini, and V. Degiorgio, Phys. Rev. Lett. 71, 4267 (1993).

[4] S. Hachisu and K. Tokano, Adv. Colloid Interface Sci. 16, 233 (1982).

[5] T. Biben and J. Hansen, J. Phys.: Condens. Matter 6, A345 (1994).

[6] G. Téllez and T. Biben, Eur. Phys. J. E 2, 137 (2000).

[7] R. van Roij, J. Phys.: Condens. Matter 15, 53569 (2003).

[8] A.-P. Hynninen, R. van Roij, and M. Dijkstra, Europhys. Lett. 65, 719 (2004)

[9] A. Philipse and G. Koenderink, Adv. Colloid Interface Sci. 100, 613 (2003).

[10] M. Rasa and A. Philipse, Nature (London) 429, 857 (2004).

[11] H. Wensink, Ph.D. thesis, Utrecht University, 2004).

[12] H. Wensink and H. Lekkerkerker, Europhys. Lett. 66, 125 (2004).

[13] M. Schmidt, M. Dijkstra, and J.-P. Hansen, Phys. Rev. Lett. 93, 088303 (2004).

[14] M. Schmidt, M. Dijkstra, and J.-P. Hansen, J. Phys.: Condens.
Matter 16, S4185 (2004).

[15] T. Biben and J. Hansen, Mol. Phys. 80, 853 (1993).

[16] L. Onsager, Ann. N.Y. Acad. Sci. 51, 627 (1949).

[17] T. Odijk, Macromolecules 19, 2313 (1986).

[18] P. Bolhuis and D. Frenkel, J. Chem. Phys. 106, 666 (1997).

[19] V. A. Baulin and A. R. Khokhlov, Phys. Rev. E 60, 2973 (1999).

[20] R. Allen, D. Goulding, and J.-P. Hansen, PhysChemComm 2, 30 (1999).

[21] S. McGrother, D. Williamson, and G. Jackson, J. Chem. Phys. 104, 6755 (1996).

[22] L. D. Landau and E. M. Lifschitz, Statistical Physics (Pergamon, Oxford, 1959).

[23] R. van Roij, M. Dijkstra, and R. Evans, Europhys. Lett. 49, 350 (2000).

[24] R. van Roij, M. Dijkstra, and R. Evans, J. Chem. Phys. 113, 7689 (2000).

[25] M. Dijkstra, R. van Roij, and R. Evans, Phys. Rev. E 63, 051703 (2001).

[26] M. P. Allen, G. T. Evans, D. Frenkel, and B. M. Mulder, Adv. Chem. Phys. 86, 1 (1993). 